\title{
LLULL ET L'ALTERITÉ CULTURELLE
}

\author{
Dominique Urvoy \\ Universidad de Toulouse
}

\section{RESUMEN:}

¿En qué medida un pensador medieval puede valer como modelo para la moderna meditación sobre la confrontación entre civilizaciones? Estudiaremos succesivamente la situación concreta del personaje frente al plano cultural de su público, su percepción de las exigencias espirituales de su auditorio, y por fin sus eventuales errores con lo que se puede deducir de ellas. Se concluye de eso la necesidad de no limitarse en el examen de las influencias textuales.

Palabras clave: Los otros, civilización, confrontación, intercomprensión, receptividad.

\section{ABSTRACT:}

In which way a medieval Thinker can be used as a Model for the modern Reflection upon the Civilizations' Confrontation? We are studying successively the Character's concretual Situation toward the cultural Background of his Public, his Perception of spiritual Needs of his Audience, and at last his possible Mistakes and its Lessons. In Conclusion, the Necessity of not limiting ourselves at the Examination of textual Influencies.

Key words: Others, civilization, comfrontation, intercomprehension, receptiveness.

La question de la confrontation avec un univers mental différent est récente. Ce n'est guère qu'au XIX siècle que l'on met en avant l'appartenance à une civilisation particulière comme déterminant des cadres de pensée. La réflexion systématique sur autrui ne commence vraiment qu'avec Husserl. Et il faudra attendre Merleau-Ponty pour que l'autrui collectif entre de plein droit dans l'analyse philosophique.

Pourquoi alors accoler le nom d'un penseur médiéval à une notion qu'il ne pouvait imaginer? Une réponse possible serait de trouver des traces prémonitoires, des anticipations qui, pour inconscientes qu'elles soient, n'apparaissent pas moins comme justifiant, a posteriori, le rapprochement avec tel auteur moderne. C'est ainsi, par exemple, qu'on a pu trouver des traces d' «intentionalité» chez des penseurs du Moyen Age arabe.

Il existe cependant une autre réponse, à laquelle nous nous tiendrons ici. C'est l'exemple que constitue le cas de Llull, cas unique en Occident d'un penseur d'envergure écrivant délibérément au moins une partie de son oeuvre à l'intention d'un public arabo-musulman. ${ }^{1}$ La question qui nous

1 Je ne reprend’s pas jci les données factuelles, déjà rassemblées dans mes deux études: Penser l'Islam. Les présupposés islamiques de l' «Ait» de Lull, Paris, Vrin, 1980, et «La place de Ramon Llull dans la pensée arabe», Catalan Review, IV, 1-2 (1990), pp. 201-220. 
occupera sera alors: en quoi consiste ce rôle d'exemple? Bien que les divers aspects soient liés entre eux, pour la clarté de l'analyse nous les sérierons en trois: 1) la situation concrète du personnage par rapport à l'arrière-plan culturel de son public; 2) sa perception des besoins spirituels de son auditoire, besoins auxquels il prétend répondre; 3) ses éventuelles erreurs et leur enseignement.

1) L'histoire de la philosophie situe généralment un auteur par rapport à d'autres auteurs par l'intermédiaire de textes, ou du moins de concepts, de problèmes, de formulations [...], qui passent de l'un à l'autre, soit pour être repris tels quels, soit pour être transformés, mais de façon à laisser transparaitre l'original. A. Koyré a montré que cela ne suffisait pas et que, pour expliquer certaines pensées, il fallait faire intervenir des courants spirituels, des mouvements sociologiques, tout ce qui est porteur de choix, de goût ou de répulsion. Bien sûr, même là il est souhaitable de disposer de textes, de façon à baliser le terrain pour éviter toute vaticination gratuite. Mais si ceux-ci sont nombreux pour les sujets étudiés par Koyré, qui appartiennent à une même aire culturelle, et qui plus est, jouissant de l'imprimerie (européens de la Renaissance et du XVII siècle), pour les questions de contact entre aires culturelles différentes, et avant Gutemberg, ils sont rarissimes. Dans notre cas, ils se réduisent aux témoignages, ouverts ou indirects, de Llull lui-même.

Pour l'un d'eux, la Logica Algazelis et son adaptation versifiée catalane, on se trouve dans la situation classique d'un texte explicitement cité, qui est simplement l'objet de remaniements. Les diverses phases en ont été analysées par Ch. Lohr. ${ }^{2}$ Mais même si, avec A. Bonner, ${ }^{3}$ on place ces livres tout à fait au début de l'oeuvre de Llull (vers 1271-2, à Montpellier), ils n'influent sur celle-ci que sur des points particuliers, comme le problème des faux arguments et la conception des Prédicables et des Catégories comme méthode d'élévation pour l'entendement, et non sur l'architecture spécifique de sa pensée et notamment la méthode de l'Art.

Pour les textes non cités mas identifiables avec une quasi-certitude (en l'occurence la partie logique du Budd al- 'rif d'Ibn Sab 'în, reconnue également par Ch. Lohr) ${ }^{4}$ on reste encore dans la démarche habituelle de définition d'une filière de transmission d'idées par des documents précis. Mais leur impact, pour être plus profond sur la pensée lullienne, n'en reste pas moins au niveau des questions de formulation assez tardive (à partir de 1293) d'une doctrine déjà élaborée antérieurement pour l'essentiel.

Les premiers travaux sur les relations de Llull avec l'Islam ont été conduits par J. Ribera et M. Asin Palacios sur la base de la référence que fait le Blaquerna aux paroles extatiques (shatahât). Ils en avaient déduit que le Llibre d'amic auquel elle introduit, devait être d'inspiration soufie. En ajoutant le thème des Cent noms de Dèu, qui renvoie au culte musulman pour les quatre-vingt-dix-neuf noms divins, et en rapprochant les figures de l'Art des figúres ésotériques que l'on trouve dans les traités de talismanique, on aboutissait à faire de Llull un «soufi chrétien».

2 Lohr, C., Raimundus Lullus' Compendium Logicae Algazelis. Quellen, Lehre und Stellung in der Geschichte der Logik, Inaugural-Dissertation, Freiburg i. Br., 1967.

3 Bonner, A., Obres selectes de Ramon Lull, Mallorca, Moll, 1989, t. II, p. 542.

4 Lohr, C., «Islamic Influences on Lull's Logic», Estudi general, 9 (1989), pp. 147-157. Tout récemment A. Bonner a proposé de compléter cette relation par des emprunts à des oeuvres d'Avicenne. Cf Bonner, A., «Possibles fonts musulmanes de les deu regles i questions de Ramon Llull», Pensar en diàleg. Miscel-lània en hommenatge al prof. $D r$. Eusebi Colomer: Revista Catalana de Teologia, XIX (1995), pp. 93-98. 
A quoi Asin Palacios, constatant que le principal soufi espagnol était Ibn Arabi, S'efforçait d'ajouter l'établissement de parallélismes entre les deux auteurs, parallélismes qu'il interprétait en outre en termes d'influence. Les Lullistes n'eurent pas de peine à démonter cet édifice, notamment du fait que le Llibre d'ami' est d'inspiration troubadouresque et non soufie, et que Llull insiste sur le thème de la possibilité d'un centième nom divin plutôt que sur un contenu spécifiquement musulman des quatre-vingt-dix-neuf autres.

En poussant l'analyse, on peut conclure que les deux références explicites aux shatahât et aux noms divins n'ont eu de valeur que publicitaire et ont visé un public purement occidental. Il s'agissait seulement de rendre celui-ci disponible, de lui montrer que le monde musulman valait la peine que l'on s'intéressât à lui autrement que militairement (les Cent noms sont offerts au Pape Nicolas IV, le premier pape franciscain).

Le résultat de ces références est ambigu. D'une part elles sont fallacieuses car n'impliquant pas, en soi, une réelle assimilation de thèmes islamiques. A la limite on peut les considérer comme de moindre portée que les conseils pratiques (sur la façon d'envisager la vie, sur les thèmes d'exhoration,[...]) qui prennent le monde musulman pour modèle. Mais d'autre part ils sont une première forme d'ouverture à l'altérité, à la différence de l'emprunt textuel qui est basé sur l'idée implicite que tout est transférable tel quel. Dans quelques cas Llull est conscient que cela pose problème. C'est ainsi que la Logica del Gatzel mentionne la notion de tawâtur, très spécifique de la conception traditionnelle du savoir en Islam. Le Llibre de contemplació emploie tel quel un autre mot arabe, celui de ramz, ce qui signifie la perception d'une nuance entre lui et le «symbole» occidental. Mais la logique du texte entraîne aussitôt l'auteur vers autre chose et il ne s'arrête pas à analyser ces hiatus qu'il a pourtant confusément perçus. Les préfaces aux Llibre d'amic et aux Cent noms de Dèu donnent davantage la possibilité de manifester ces spécificités. Mais les aperçus qu'elles donnent sont contrés aussitôt par la formation troubadouresque de l'auteur d'une part, par sa volonté apologétique de l'autre, encore que, dans ce dernier cas, il y ait plus de conséquences qu'une vision superficielle des choses ne le laisserait croire.

Il y a, en effet, des éléments qui sont d'une importance capitale pour l'esprit même de la philosophie lullienne, sans que l'auteur signale qu'ils ont un correspondant en Islam, et encore moins qu'il puisse s'agir d'emprunt de sa part. L'un est le choix des dignitates. Celles-ci étant présentées dans les diverses rédaction de l'Art, sous la forme d'une axiomatique minimale, et se retrouvant dans la liste des Cent noms de Dèu (écrite à Rome en 1288), on en a tiré argument contre l'idée de toute emprise islamique sur celui-ci. Mais si on regarde la question dans l'autre sens on constate que jusque dans cet ouvrage relativement tardif par rapport aux premières formulations de l'Art on voit les concepts spécifiquement lulliens s'insérer dans un ensemble parfaitement admissible par un théologien musulman.

$\mathrm{Si}$ on compare, en effet, la liste lullienne des noms divins avec les listes couramment admises en Islam, on constate que vingt-huit de la première ont leur exacte traduction dans la seconde et que, si on ajoute les synonymes et les variantes dans l'interprétation ou des sens allégoriques couramment admis, on atteint environ la soixantaine. Or le Kitâb al-Irshâd de Juwaynî, oeuvre la plus répandue en Andalus, n'envisage que quatre-vingt noms, et les termes qu'il délaisse sont ceux qui concernent les rapports de Dieu à Ses créatures (péché, grâce, ...), c'est-à-dire les mêmes que ceux que Llull ignore, pour les remplacer par des termes propres au Christianisme ou à l'Art. Bien plus, 
malgré les transformations apportées, la liste lullienne conserve la même répartition que dans la théologie ash 'arite: l'essence de Dieu, Ses actes, Ses attributs éternels.

Un autre aspect caractéristique à noter est que Llull est - semble-t-il - le premier à employer le terme "théologie positive". Or on sait que le Kalâm a élaboré la distinction entre ce qui relève de la raison ('aqliyât) et ce qui relève de la tradition (sam iyât), rejetant la première catégorie du côté de la philosophie, et définissant la seconde comme «sciences positives de la Loi religieuse» (al-'ulûm al-shar'iyat al-wad'ciya). Cette distinction a été largement popularisée par l'Encyclopédie des Fréres de la Pureté qui, bien que chiite d'origine, a été diffussé dans tout le monde arabe sunnite jusqu'au XIVe siècle. ${ }^{5}$

On voit par là qu'il y a une sorte de renversement en passant des références de Llull à la civilisation islamique à son impact réel sur lui. S'il cite expressément un texte, c'est de peu de signification culturelle. S'il utilise des textes sans les citer, cela a une valeur surtout pragmatique. S'il fait appel nommément à des pratiques cultuelles, c'est déjà une ouverture, mais c'est surtout pour se donner une stature particulière auprès du public européen. Enfin l'analyse décèle des points communs fondamentaux avec la culture musulmane, que Llull n'éprouve pas le besoin de noter. Il n'y a pas de raison d'invoquer pour cela un réflexe de prudence, car cela aurait dû jouer plutôt pour les shatahât. Il semble simplement que cela lui parât tout naturel de tabler sur un terrain commun avec l'interlocuteur potentiel.

Ici nous sommes en dehors de toute problématique d'influence au sens habituel du terme, qui renvoie à une sorte de transitivité des idées. Si influence il y a c'est celle d'un contexte de confrontation. Confrontation à divers niveaux: à l'intérieur même de l'Islam majorquin, que Llull vise par priorité, il y a eu le conflit entre Ash'arism' et Zâhirisme, conflit ravivé par la brève présence almohade juste avant la conquête chrétienne et la naissance de notre auteur. Face à l'idiosyncrasie ainsi formée, Llull a choisi de se référer de préférence à la tradition chrétienne qui lui paraissait la plus susceptible d'être en prise sur elle, et c'est la tradition anselmienne des rationes necessariae.

Il n'y a là rien de mécanique ni de transitif. Il y a par contre l'intuition de climats intellectuels, intuition qui détermine les choix conceptuels.

2) On s'est souvent extasié sur les nombreuses innovations introduites par Llull dans la pensée européenne, que ce soit dans la forme ou dans l'expression. Cette réaction est souvent due à une vision strictement européocentrique des choses. Llull appartient aux deux mondes européen et méditerranéen. T. et J. Carreras i Artau avaient à juste titre choisi de le désigner comme «penseur de frontière», mais n'étant pas eux-mêmes arabisants, et peut-être aussi rendus méfiants par les errances des arabisants de leur temps déjà évoquées plus haut, ils n'en avaient pas tiré toutes les conséquences.

Or sur bien des points on s'aperçoit que ce qui était début en Europe, avait déjà une histoire dans le monde méditérranéen. Cette idée répugne à beaucoup, qui ne pensent - encore une fois qu'en termes positivistes d'influence textuelle. Mais dire que si Llull est le premier en Europe à user de la langue vulgaire pour exprimer des thèmes philosophiques, il s'inscrit par contre au sein d'un vaste mouvement dans le cadre de la Méditerranée, mouvement qui commence avant lui, en

5 Cf Lohr, C., Leçon d'investiture comme Magister de la Maioricensis Schola Lullistica, Estudios lulianos, XVII, 1 (1973), pp. 114-127. 
Espagne même (Shushtarî surtout, mais également un peu Ibn Arabî), d'où il s'étend à l'Egypte, et qui continue après lui en s'élargissant à des pays non arabes comme la Turquie, ne signifie pas qu'il copie tel ou tel, ni même qu'il l'imite consciemment. Cela veut dire simplement qu'il perçoit dans son public le même besoin qu'ont perçu ceux qui ont initié le mouvement et que percevront ceux qui le prolongeront. Dans ces transferts il faut plutôt penser à des véhicules d'idée de type esthétique (comme la musique qui, tant qu'elle est restée monodique, a été moins cloisonnée en aires de civilisations qu'après la généralisation de la polyphonie) qu'aux écrits eux-mêmes.

J'ai employé, pour désigner cela, l'expression: «une sorte de perception sensitive des tendances diffuses dans le monde arabo-musulman», ${ }^{6}$ expression de d'aucuns on trouvé «très peu convaincante». ${ }^{7} \mathrm{~J}$ 'admets fort bien qu'elle laisse insatisfait, encore qu'elle corresponde à ce que l'on utilise en histoire des sciences pour expliquer l'apparition simultanée, chez deux savants indépendants l'un de l'autre, d'une même découverte. Mais je suis, pour ma part, encore plus insatisfait de voir que l'on trouve normal d'une part de réserver à Lull le miracle d'être un commencement absolu, et de l'autre qu'on ne puisse le faire qu'en décidant arbitrairement de faire l'impasse sur tout le contexte méditerranéen. Les historiens - notamment à la suite de $\mathrm{M}$. Lombard — ont su surmonter l'erreur de H. Pirenne qui établissait, à partir de la conquête musulmane, une frontière infranchissable entre Nord et Sud de la Méditerranée, pour reconnaître les multiples échanges qui ont continue à avoir lieu; les historiens de la philosophie -et ce malgré tous les efforts d'un A. de Libéra, qui n'est pourtant pas un arabisant et ne peut être soupçonné de «prêcher pour sa paroisse»— semblent avoir beaucoup de peine à les suivre.

La question est simple. Llull répète qu'il veut parler aux non-Chrétiens et notamment aux Musulmans. Ses textes affirment à plusieurs reprises qu'il a écrit d'abord en arabe des livres dont nous n'avons qu'une version catalane ou latine ultérieure (le Llibre del Gentil, une partie au moins du Libre de contemplacio, le Liber de Trinitate et Incarnatione, la Disputatio Raimundi Christiani et Hamar Sarraceni, l'Ars consilii), ou qu'il a traduit dans la langue de l'Islam des textes déjà composés en latin ou catalan (l'Ars inventiva veritatis, le Dictat de la Trinitat, et peut-être le Llibre del Es de Dèu). Est-ce un mensonge? Les témoignages, comme celui du Cancionero de Ixar, qui prétendent que ces textes ont bien existé et circulé au Maghreb sont-ils aussi des affabulations? ${ }^{8}$

6 Urvoy, D., «Nature et portée des liens de Ramon Lull avec l'univers arabe», dans Aristotelica et Lulliana, F. Dominguez, R. Imbach, T. Pindl et P. Walter éd., la Haye, Martinus Nijhoff International, 1995, p. 427.

7 Santanach, J., compte-rendu du précédent, Studia lulliana, XXXVI (1996), p. 169.

8 Certains, comme A. Llinarès, nient la réalité de ces textes, en arguant qu'il faudrait qu'«on les montre». C'est oublier que beaucoup d'écrits ont disparu, faute d'être recopiés, sans que l'on remette en question leur existence attestée par des témoignages. D'Averroès nous ne disposons en arabe que de deux de ses grands commentaires d'Aristote et des fragments d'un autre. Est-ce à dire que ces livres que nous n'avons qu'en hébreu ou en latin ne sont que ce que les Anglais appeleraient des «forgeries»? D'autres textes, qui n'ont pas été traduits - notamment les textes almohades - ne sont connus que par le titre. Est-ce à dire que les listes anciennes qui les mentiennent mentent? Personne, que je sache, ne conteste la légitimité de l'entreprise d'E. Bignone, par exemple, s'efforçant de reconstituer «l'Aristotele perduto». Pourquoi refuser la légitimité d'une reconstitution d'un «Llull arabe»?.

En 1971, le P. Valverde O.F.M., vivant en Algérie, se présenta au chanoine S. Garcias Palou, alors recteur de la Maioricensis Schola Luilistica. Il lui lut la traduction de textes qu'il disait avoir trouvés dans des bibliothèques algériennes. S. Garcias Palou, parfait connaisseur des oeuvres de Llull, en élimina certains, mais considéra que d'autres ne pouvaient avoir été écrits que par Llull lui-même, et pas seulement par un quelconque Arabe chrétien comme le lui suggérait son interlocuteur. Malheureusement, lorsque le recteur raconta lui-même cette histoire au chercheur débutant que j'étais alors, le P. Valverde avait quitté les ordres et n'avait plus donné signe de vie, ce qui m'empêchait de préciser l'origine des documents. 
Mais si on accepte la réalite de ces textes arabes, il faut se demander aussitôt s'ils ont eu un sens. Llull a-t-il parlé pour être compris, ou n'était-il qu'un monomaniaque qui écrivait sans se soucier de ses interlocuteurs?

$\mathrm{Si}$ on suppose une volonté d'efficacité -comme on le fait maintenant, à la suite de J. Hillgarth, pour expliquer son action auprès des responsables politiques et religieux de son tempson constate que son écriture s'insère dans des procédés qui peuvent éventuellement avoir déjà connu en Occident des balbutiements, mais qui ont été largement développés dans la civilisation arabe. $^{9}$

Tel procédé est destiné à enrichir la forme de pensée occidentale. C'est le cas en particulier des «corrélatifs». De l'aveu de Llull lui-même, ils sont calqués sur «la façon de parler arabe», C'est à dire sur l'usage des flexions internes qui, dans une langue indo-européenne, sont remplacées par des jeux de suffixes (are, ans, atus, abile, [...]). De même que les auteurs arabes, surtout depuis Fârâbi, ont privilégié les trois formes du masdar, du fấil et du maf' ûl, ce qui a permis aux Chrétiens (Yahyâ ibn 'Adî, Ibn al-Tayyib,[...]) d'en faire une «démonstration» de la trinité, de même Llull retrouve cette démarche et l'exploite avec une certaine naiveté car il semble ignorer les réfutations que cela a suscitaé à l'interieur de l'Islam.

Tel autre procédé a manifestement pour but d'avoir prise sur l'interlocuteur arabe. C'est le sens de la combinatoire, à condition d'oublier les comparaisons superficielles qui ont été faites entre les figures circulaires que l'on peut trouver de part et d'autre, car d'une part, au-delà des cercles qui sont l'élément commun, il y a de nombreuses différences, et de l'autre certaines expressions de l'Art sont dépourvues de figures.

Il faut donc replacer celles-ci dans une logique d'ensemble. Or dans l'univers arabe on peut suivre toute une évolution qui, des traductions d'Aristote jusqu'au XIII ${ }^{\mathrm{e}}$ siècle, précisément, met en place les éléments d'une mécanique combinatoire: les modes de formation du réel par combinaison d'éléments plus simples, admis par la plupart des Grecs, est théorisée par Aristote en quatre procédures essentiellement qualitatives; le monde arabe les traduit en insistant sur la combinaison chimique et en favorisant la manipulation; l'alchimie s'empare de cela et l'amalgame à la technique sémitique ces combinaisons de lettres (sîmiy $\hat{a}$ ), aboutissant aux représentations par «cases»; l'idée d'un privilège du mouvement circulaire amène à mettre en avant des représentations par cercles qui, progressivement, évoluent de la représentation de «domaines» à celle de «place en vue d'une mise en correspondance avec d'autres élements du même cercle ou d'un autre cercle concentrique».

On peut néanmoins conclure deux choses. D'une part il existe très vraisemblablement en Afrique du Nord des textes arabes lulliens, au moins à l'état de fragments. De l'autre,ces textes ont de fortes chances de n'être conservés que dans des miscellanea, ce qui expliquerait que l'on n'ait pas de titre clair apparaissant dans les catalogues de bibliothèques. Il reste à attendre patiemment qu'un chercheur assez curieux, et - s'ii est musulman - d'esprit assez ouvert pour ne pas mépriser des productions chrétiennes, ait le courage de refaire les recherches nécessaires.

9 Cf Urvoy, D., «Les Musulmans pouvaient-ils comprendre l'argumentation lullienne?», Estudi general, 9 (1989), pp. 159-170; «Sur un aspect de la combinatoire arabe et ses prolongements en Occident», Arabica, XXXIX (1992), pp.25-41; «Le symbole de l'arbre chez les auteurs arabes antérieurs à Luil», dans Constantes y fragmentos del pensamiento luliano, F.Dominguez et J. de Salas éd., Tubingen, Niemeyer, 1996, pp. 91-97; et les travaux déja cités. Sur l'attitude pratique de Liull envers le monde arabe, of «L'idée de Christianus arabicus», Al-Qantara, XV, 2 (1994), pp. 497-507. 
L'Art lullien suppose une reinterpretation de cela, et surtout, quand il met en jeu des représentations graphiques, une «répartition» des fonctions de la figure circulaire selon qu'elle représentait, chez les Arabes, une trajectoire des êtres ou des esprits revenant sur son origine (remplacée par l'ascensus et descensus intellectus), un domaine ferme (les camerae et les cercles du Vrai et du Faux de l'Art), ou le support de mises en correspondance, prolongeant l'image de la rotation de la sphère du monde, génératrice de mixtion (les figures patientes et les figures agentes de l'Art).

3) Mais Llull n'est pas seulement au contact du monde arabe. Issu des milieux de la conquête, et surtout cherchant ses appuis dans des milieux qui ne sont pas portés au dialogue des cultures (cours royales, cours pontificales, université de Paris), il est tiraillé entre l'attitude d'ouverture et celle du repli intransigeant.

Cela peut aboutir à une véritable schizophrénie, dont on voit un exemple particulièrement éloquent dans un opuscule écrit à Majorque en février 1313, à l'usage des commerçants allant en pays musulmans. A ces missionnaires de circonstance, peu informés de leur propre foi, et qui peuvent être tentés par l'Islam, Llull entend prouver que celui-ci est «bon» mais «incomplet». Le Prophète Muhammad a dit des choses erronées et impies sur le Christianisme: négation de la Trinité, de l'Incarnation, de la Crucifixion et de la résurrection; mais beaucoup de ses dires également coïncident avec ce dernier: Dieu créateur de toutes choses; Jésus-Christ esprit et verbe de Dieu, né de la vierge Marie, vivant et monté au ciel, et qui est le meilleur des hommes de tous les temps; sainteté des douze apôtres. Or Llull a donné deux rédactions de ce texte. ${ }^{10}$ Dans l'une, le ton envers le Prophète est neutre, mais dans l'autre, Llull parle du «poison» de Muhammad, qui «obnubile» la «secte perverse» de ses adeptes; le Prophète lui-même est qualifié d' «apostat».

Dans ce cas on ne peut guère invoquer que des facteurs personnels. Dans d'autres, ce sont vraiment des questions de confrontation de civilisations qui sont en jeu. C'est le cas, par exemple, de la différence qui saute aux yeux entre la présentation de l'Islam que fait la Doctrina pueril et celle du Llibre del gentil, qui semblent pourtant relever de la même période (entre 1274 et 1276). ${ }^{11} \mathrm{La}$ première correspond tout à fait aux stéréotypes polémiques, alors que l'on a toujours remarqué le ton irénique et l'effort d'objectivité du second.

La raison en est que l'un se rattache à la littérature européenne, étant directement écrit en catalan, alors que le second a eu une première rédaction en arabe, qui a mis Llull en relation avec un ensemble d'idées circulant dans le monde arabe Comment a-t-il connu ces idées? Plus tard il citera nommément deux oeuvres de polémique christiano-musulmane arabes: la Risâla d'al-Kindî le Nestorien, bien connue chez les Mozarabes d'Espagne, et la Contrarietas alfolica, vraisemblablement altération d'Ikhtilâf al -fuqqahâ'. Ces oeuvres, si ce sont les seules qu'il a connues, se font partiellement l'écho d'un mouvement de contestation-réflexion sur le fait religieux, mouvement qui a couvert tout le monde musulman de l'extrême-Est jusqu'en Andalus, et qui a eu également des répercussions dans le monde des falâsifa. ${ }^{12} \mathrm{Ce}$ mouvement commence par greffer sur un stock

10. Liber per quem poterit cognosci quae lex sit magis bona, magis_magna et etiam magis vera, et Liber per quem potest discernere quae lex melior, maior et clarior ac verior habeatur; édité par M. Senellart, dans les textes de 1312-1313: Raimundi Lulli Opera Latina; Corpus Christianorum, continuatio medievalis, Turnhout, Brepols.

11 Cf Bonner, A., Obres selectes de R. L., o.c., t. II, pp. 543-4.

12 Sarah Stroumsa, de l'Université hébraique de Jérusalem, qui a étudié les zanâdiqa (hérétiquès musulmans), travaille actuellement sur ce sujet. 
d'objections, déjà ancien mais toujours croissant, une interrogation sur le «pourquoi?» de la croyance religieuse. Il s'oriente ensuite succesivament vers l'analyse objective des croyances mais dans un but d'examen strictement logique (al-Warrâq, IX ${ }^{e}$ s.), la prise en considération des intentions des croyants (al-Arfadî, XI ${ }^{\mathrm{e}}$ s.) et enfin le libre exposé par chacun de la foi à laquelle il adhère, avec tous ses aspects tant intellectuels que sentimentaux (Ibn Kammûna XIII ${ }^{\mathrm{e}}$ s.). Llull, qui écrit quelques années avant ce dernier et qui ne semble avoir eu aucune relation avec cet auteur juif de Bagdad, n'en répond pas moins aux mêmes visées et exigences que lui, lesquelles sont l'aboutissement de tout le processus évoqué. ${ }^{13}$

Or le Llibre del gentil n'aura pas de suite, le Liber de quinque sapientibus, par exemple, prenant une allure très démonstrative et apologétique, dans le droit fil de tout le reste de l'oeuvre lullienne. Il y a donc eu de sa part soit des renoncements conscients, soit des oublis.

Le plus caractéristique est celui qui concerne Averroès. Le penseur catalan ne semble pas le connaître directement. Il ne le voit qu'à travers la condamnation de l'évêque de Paris, Etienne Tempier. Non seulement il n'utilise - pas comme le fait son compatriote Ramon Marti, dont il conteste, par ailleurs, la démarche - les textes non traduits en latin, et notamment les textes théologiques où Averroès parle en son nom propre, mais il se fait l'écho de légendes erronées, comme celle selon laquelle l'auteur cordouan aurait été lapide pour ses erreurs par ses coreligionnaires.

A quoi on peut ajouter que cela explique pourquoi l'étude de la Logique de Ghazâlî a eu si peu d'impact sur lui. Cet auteur n'a été connu dans l'Occident latin que par ses Maqâsid al-falâsifa, exposé objectif des opinions des philosophes orientaux, mais non la réfutation du Tahâfut-al-falâsifa, faite du point de vue religieux, qui lui fait suite. En travaillant sur le texte des Maqâsid, Llull a cru avoir affaire à un de ces auteurs qu'on jugeait, à l'époque, transférable tels quels d'un point à l'autre du globe. D'où son désintérêt pour des notions spécifiquement islamiques comme tawâtur, lors même qu'il les relève.

On voit par là que la question des influences textuelles n'est qu'un aspect -certes non négligeable, mais un aspect seulement- des liens entre Llull' et le monde arabe, qui est un problème beaucoup plus complexe. C'est précisément en tant qu'il est révélateur de cette complexite que Llull nous apparait comme pouvant servir de modèle pour la réflexion d'aujourd'hui.

Dominique URVOY Université de Toulouse II 5 , allées Antonio Machado 31058 Toulouse. Cedex France

13 Cf Urvoy, D., Les penseurs libres dans l'Islam classique, Paris, Albin Michel, 1996. 